\title{
Spectroscopic Measurements in a Titanium Vacuum Arc with Different Ambient Gases
}

\author{
D. Grondona, H. Kelly*, M. Pelloni, and F. O. Minotti* \\ Instituto de Física del Plasma (CONICET), Departamento de Física, \\ Facultad de Ciencias Exactas y Naturales (UBA) Ciudad Universitaria Pab. I, (1428) Buenos Aires, Argentina
}

Received on 30 December, 2003; revised version received on 28 April, 2004

\begin{abstract}
Spectral emission lines from a low pressure d-c arc discharge with a Ti cathode were studied in various ambient gases; $\mathrm{N}_{2}, \mathrm{O}_{2}$, and Ar. Light from the plasma was detected by an optical spectrometer multichannel analyzer (OSMA). The spectral intensities of $\mathrm{Ti}, \mathrm{Ti}^{+}, \mathrm{Ar}$ and $\mathrm{N}_{2}$ were measured as a function of the gas pressure in the range $5 \times 10^{-3}-0.5$ mbar. The measurements were performed in the inter-electrode region at different distances from the cathode. For $\mathrm{N}_{2}$ and $\mathrm{O}_{2}$ as the filling gases, the intensities of $\mathrm{Ti}$ and $\mathrm{Ti}^{+}$increase with the gas pressure up to pressure values of the order of $0.2-0.4 \mathrm{mbar}$, while they decrease for higher pressure values. With Ar gas, a different behavior of the $\mathrm{Ti}^{+}$intensity was found; it presents an increasing general trend. The behavior of the lines was qualitatively analyzed in terms of the most relevant atomic processes that take place in the metallic plasma - gas structure (charge-exchange, electron impact excitation and ionization, etc.). It is found that the behavior of the observed spectral lines can be satisfactorily explained in terms of the relevance of these processes as functions of the neutral gas density and electron temperature.
\end{abstract}

\section{Introduction}

In a vacuum or low-pressure arc a plasma jet with energetic metallic ions $(20-100 \mathrm{eV})$ is ejected from the cathode surface. Under vacuum operation, these ions can be deposited onto any substrate located in the inter-electrode space, forming a metallic coating[1]. In many situations, the discharge chamber is filled with a gas, either with the purpose of forming complex coatings (using reactive gases)[2] or to produce a pretreatment of the substrate surface that improves film adhesion. In particular, inert gases like argon have been used for this last purpose[3].

Several interactions of the metallic plasma jet with the gas particles take place in the discharge chamber such as elastic scattering, leading to ion slowing down and diffusion, and charge exchange reactions. Ionization of gas particles by electron impact and several recombination processes (dissociative or three body recombination) also contribute to generate a complex plasma-neutral gas structure[4].

In a previous work[5], optical emission spectroscopy was used to obtain information on this structure. $\mathrm{A} \mathrm{Cu}$ cathode was employed with $\mathrm{O}_{2}$ and $\mathrm{N}_{2}$ as background gases in the pressure range of $10^{-4}-0.4$ mbar.

In this work, a new spectroscopy study of a vacuum arc with $\mathrm{Ti}$ cathode is presented, but using also a monoatomic gas (Ar) besides $\mathrm{O}_{2}$ and $\mathrm{N}_{2}$ as filling gases, in a pressure range of $5 \times 10^{-3}-0.5$ mbar.

The behavior of the observed spectral lines can be explained in terms of the relevance of the different atomic processes that take place in the plasma inter-electrodic region as functions of the neutral gas pressure and electron temperature.

\section{Experimental setup}

The experimental setup is shown schematically in Fig. 1. The vacuum chamber is a stainless steel cylinder with a length of $40 \mathrm{~cm}$ and a diameter of $25 \mathrm{~cm}$, acting as the grounded anode. The cathode is a Ti cylinder with a diameter of $5.6 \mathrm{~cm}$, surrounded by a floating shielding, connected to the negative terminal of the arc power supply. For optical diagnosis two circular optical windows with a diameter of 4.0 $\mathrm{cm}$ were mounted on tubes with lengths of $10 \mathrm{~cm}$ protruding from the lateral wall of the chamber. The two windows (A and $\mathrm{B}$, respectively) are located at axial distances of 9 and $25 \mathrm{~cm}$ from the cathode surface.

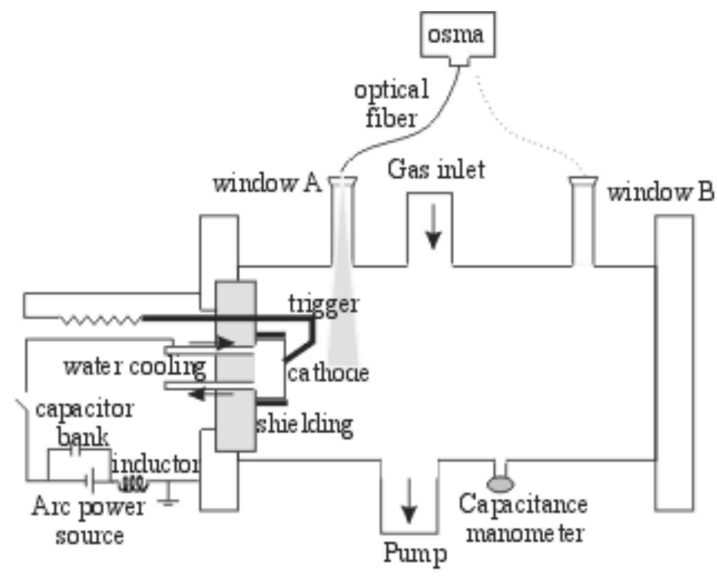

Figure 1. Scheme of the experimental setup including the spectroscopic diagnostic system.

${ }^{*}$ Member of the CONICET 
Using an oil diffusion pump the vacuum chamber is evacuated to a base pressure below $10^{-4}$ mbar and, during the discharge, a gas flux is introduced and its pressure monitored with a capacitance manometer. $\mathrm{Ar}, \mathrm{N}_{2}$ and $\mathrm{O}_{2}$ are used as filling gases in the pressure range $5 \times 10^{-3}-0.5$ mbar.

The discharge circuit consists in a current supply of 150 A and $18 \mathrm{~kW}$, in parallel with a capacitor bank of $165 \mathrm{mF}$ connected to the electrodes through a series inductor of 2.8 $\mathrm{mH}$ to provide arc stability. The discharge is ignited with a Tungsten striker put into contact (and later removed) with the cathode, and the arc is operated in a continuous mode at $100 \mathrm{~A}$.

The light emitted by the plasma is transported from the optical windows to the entrance slit of the spectrograph by optical fibers with a diameter of $1.5 \mathrm{~mm}$. This spectrograph has a crossed Czerny-Turner design with an optical grating of 1200 lines $/ \mathrm{mm}$, and the emission spectrum was monitored in the spectral region from 320 to $850 \mathrm{~nm}$. The light from the spectrograph is intensified by a multi-channel plate and detected by a photodiode array (OSMA). The sensibility of the OSMA was determined by employing a He-Ne laser of known output power as the light source. It was found that $130 \pm 20$ photons passing through the entrance slit of the spectrograph produce one count in the detector[5].

\section{Results}

In Fig. 2 are presented typical spectral diagrams obtained from window $\mathrm{A}$ for a high vacuum (pressure below $10^{-4}$ mbar) discharge. Both figures correspond to the same discharge but with different spectral ranges. The exposure time of the detector was $\sim 1 \mathrm{~s}$, and the reproducibility was good, with statistical dispersion below $15 \%$, evaluated after 15 registers obtained under identical conditions of the discharge. As can be seen, several lines of Ti-I and Ti-II are registered, but none of Ti-III (within the whole studied spectral range) in spite of being this species the most abundant in Ti cathode arcs ( $\mathrm{Ti}$ ions are emitted from the cathode spot with charge state of $z=+1,+2,+3$ and percentages of $27 \%, 67 \%$ and $6 \%$, respectively[6]).

Figure 3 shows a typical spectral diagram from window A for Ar at $(5.0 \pm 0.5) \times 10^{-2}$ mbar. Only lines from neutral Ar (Ar-I) were found.

In Fig. 4, a typical spectral diagram from window $\mathrm{A}$ is shown for $\mathrm{N}_{2}$ at $(2.7 \pm 0.5) \times 10^{-2}$ mbar. The diagram shows the characteristic band emission of neutral $\mathrm{N}_{2}$. Again, no ionized gas species were detected. Besides, no lines (or bands) corresponding to oxygen were found.

Figure 5 shows the behavior with pressure of characteristic lines of Ti-I (468.19 nm), Ti-II (454.96 nm), and Ar-I $(811.53 \mathrm{~nm})$ when $\mathrm{Ar}$ is used as filling gas. The intensity of each species (that is given in detector counts) is normalized to its maximum. Fig. 5a) shows the results from window A and Fig. 5b) those from window B. The maximum of Ti-I for window A, corresponds to approximately 0.1 mbar, while for window $\mathrm{B}$ this maximum appears at about $3 \times 10^{-2}$ mbar. The behavior of the Ti-II line from window A shows a continuous increase, while for window B it shows some oscillations but with an increasing general trend. The Ar-I lines follows approximately the Ti-I line behavior with pressure, but with maxima positions somewhat shifted.
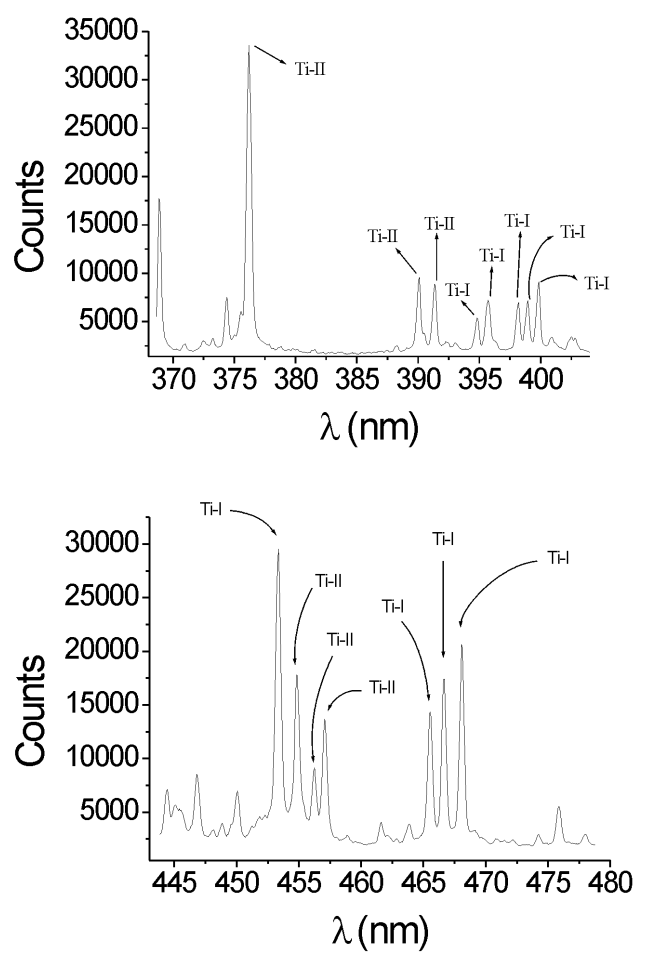

Figure 2. Spectral diagrams from window A for a dischrage in vacuum (pressure below $10^{-4} \mathrm{mbar}$ ).

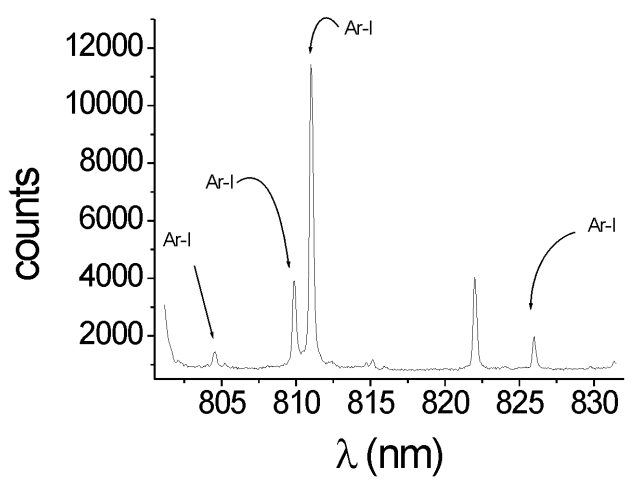

Figure 3. Spectral diagram from window A for Ar at $(5.0 \pm 0.5) \times$ $10^{-2}$ mbar. 


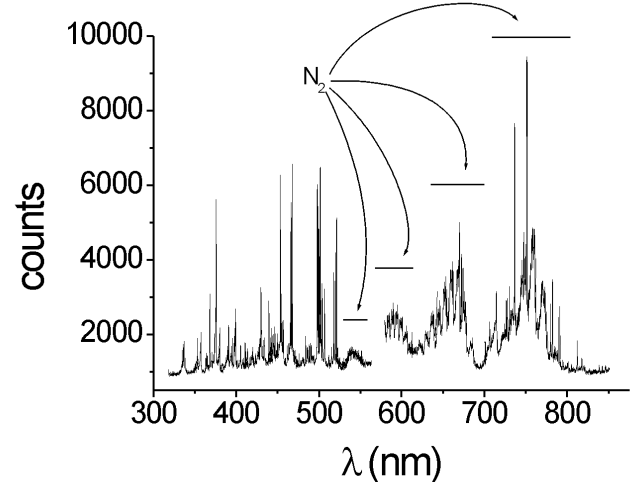

Figure 4. Spectral diagram from window $\mathrm{A}$ for $\mathrm{N}_{2}$ at $(2.7 \pm 0.5) \times$ $10^{-2}$ mbar.

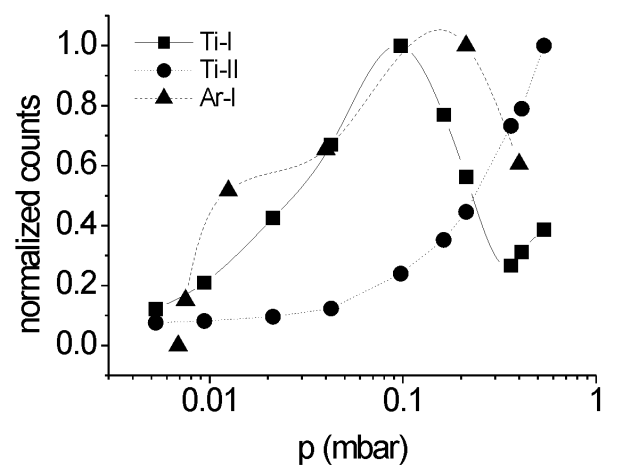

A)

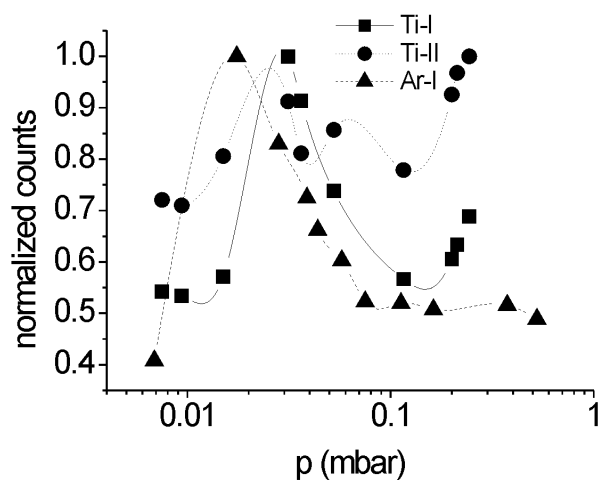

B)

Figure 5. Behavior with pressure of characteristic lines of Ti-I, TiII, and Ar-I when Ar is used as filling gas: a) results from window $\mathrm{A} ; \mathrm{b})$ results from window $\mathrm{B}$.

Figure 6 shows the behavior with pressure of the same characteristic lines of Ti-I and Ti-II used before, for window A only and for the other two gases. Fig. 6a) corresponds to $\mathrm{N}_{2}$ and Fig. 6b) to $\mathrm{O}_{2}$. From Fig. 6a) it can be seen that both $\mathrm{Ti}$ lines present the same behavior with the pressure with a maximum at $\sim 0.2$ mbar for $\mathrm{N}_{2}$, while that maximum appears at $\sim 0.3 \mathrm{mbar}$ for $\mathrm{O}_{2}$ (see Fig. 6b)). A characteristic emission line of $\mathrm{N}_{2}(775.3 \mathrm{~nm})$ is also plotted in Fig. 6a), and presents an increasing behavior at low pressure, reaching a maximum at $\sim 0.08$ mbar and following the behavior of both Ti lines.

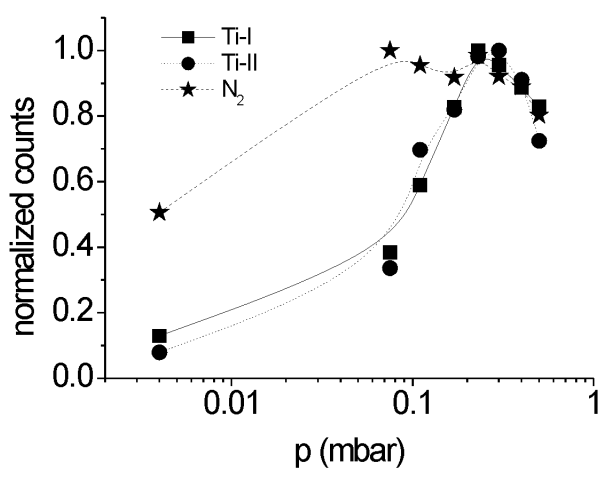

A)

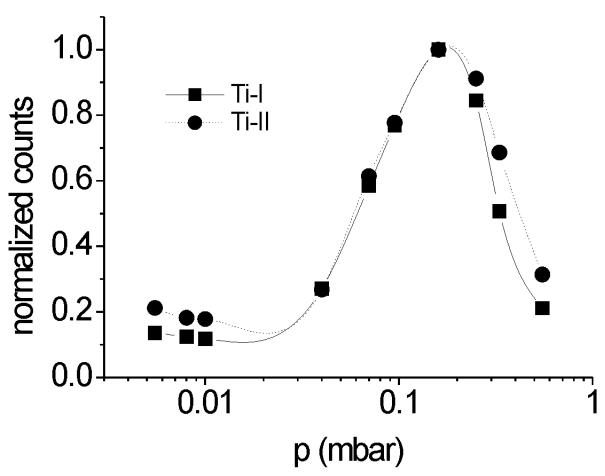

B)

Figure 6. Behavior with pressure of characteristic lines of Ti-I and Ti-II for window $\mathrm{A}$ :a) filling gas $\mathrm{N}_{2}$; b) filling gas $\mathrm{O}_{2}$.

\section{Discussion and final remarks}

A systematic study of $\mathrm{Ti}$ emission lines in the presence of various ambient gases at different pressure values has been carried out.

In order to interpret the obtained results some points are worth remarking.

Since the sensitivity of the OSMA is known, it is possible to evaluate the density of Ti excited states to obtain a given registered amplitude. Taking into account that the plasma is transparent for the studied lines[7], and apart from geometrical factors, the line intensity is proportional to the emission transition probability of the line $\left(\sim 10^{8}\right.$ $\mathrm{s}^{-1}$ ) and to the population of the upper excited state of the line. For our geometrical setup, a density of excited species 
$\sim 10^{5}-10^{6} \mathrm{~cm}^{-3}$ is enough to give an intense register (close to saturation) in the detector.

Since the ejection velocities of the ions at the spot are $\sim 10^{6} \mathrm{~cm} / \mathrm{s}[6]$, and taking into account the above quoted emission probability, the resulting mean free path for spontaneous radiative decay is $\sim 10^{-2} \mathrm{~cm}$. Hence, it can be concluded that the generation of a given excited state population is a local phenomenon which has no memory of the original ion state at the spot.

The absence of Ti-III lines, in spite of the fact that $z=+2$ is the most abundant species for a Ti cathode, can be explained considering that charge-exchange cross sections are very high, so practically no Ti-III species can be found at window A even for very low gas pressures[4]. Besides, the absence of this line is also consistent with the lack of excited particle levels by electron impact, since visible transitions of Ti-III correspond to highly excited levels (with bound energies of about $20 \mathrm{eV}$ ) while the electron temperature in the inter-electrode space is $\sim 1.8 \mathrm{eV}$ (or less, depending on the gas pressure value)[8], too low for that ion excitation energy level.

When Ar is used as filling gas, the Ti-I line increases at low pressure values, while decreases at higher values. The increase can be explained in terms of the neutral Ti species being populated by charge-exchange from higher chargestate species with Ar gas (in fact the maximum of Ti-I line coincides approximately with the pressure which makes the mean free path for charge-exchange of the order of the window position; note that window $\mathrm{B}$, that is located at a distance three times larger than window A presents the maximum of Ti-I line at a pressure value three times lower). The decrease at high pressure can be explained because chargeexchange has been exhausted and neutral $\mathrm{Ti}$ is excited by electron collisions, but with electrons progressively colder. The Ti-II line shows a continuous increase with pressure, which can be explained by charge-exchange from Ti-III (at low pressure) and by charge-exchange of $\mathrm{Ar}^{+}$ions with neutral Ti. The behavior of the Ar line with pressure follows approximately the behavior of the electron temperature.
In the presence of molecular gases, there are practically no gaseous ions (because of the presence of dissociative recombination, which gives a strong remotion channel for gaseous ions), and hence the lines follow the behavior of the electron temperature.

To summarize, the registered behavior of the spectral lines can be explained in terms of the relevance of the different atomic processes that take place in the plasma interelectrodic region as function of the neutral gas pressure and the electron temperature.

\section{Acknowledgments}

This work was supported by a grant from the Buenos Aires University (PID X214) and from the Agencia Nacional de Promoción Científica y Tecnológica (PICT 03-09491).

\section{References}

[1] R. L. Boxman, D. M. Sanders, and P. J. Martin, Handbook of Vacuum Arc Science and Technology, Fundamental and Applications, Park Ridge, NJ: Noyes, 1995.

[2] H. Randhawa, Thin Solid Films 167, 175, 1988.

[3] C.A. Davis, M. K. Puchert, D. R. McKenzie, and D. W. James, Proc. XVth Int. Symp. on Discharges and Electrical Insulation in Vacuum, Darmstadt, 1992, 690.

[4] A. Lepone and H. Kelly, J. Phys. D: Appl. Phys. 34, 3043, 2002.

[5] D. Grondona, H. Kelly, A. Márquez, and A. Lepone, IEEE Trans. Plasma Sci. 30, 391, 2002.

[6] J. Kutzner and H. C. Miller, IEEE Trans. Plasma Sci. 17, $688,1989$.

[7] W. Lochte-Holtgreven, Plasma Diagnostics, New York:AIP, 1995.

[8] A. Lepone, H. Kelly and A. Márquez, J. Appl. Phys. 90, 3174,2001 